\title{
Fast and Easy Quantitative Characterization of Methanotroph-Photoautotroph Cocultures
}

\author{
Kiumars Badr ${ }^{1}$, William Whalen ${ }^{1}$, Q. Peter $\mathrm{He}^{1}$, and Jin Wang ${ }^{1}$ \\ ${ }^{1}$ Auburn University
}

August 10, 2020

\begin{abstract}
Recent research has demonstrated that synthetic methanotroph-photoautotroph cocultures offer a highly promising route to convert biogas into value-added products. However, there is a lack of techniques for fast and accurate characterization of cocultures, such as determining the individual biomass concentration of each organism in real-time. To address this unsolved challenge, we propose an experimental-computational protocol for fast, easy and accurate quantitative characterization of the methanotroph-photoautotroph cocultures. Besides determining the individual biomass concentration of each organism in the coculture, the protocol can also obtain the individual consumption and production rates of $\mathrm{O} 2$ and $\mathrm{CO} 2$ for the methanotroph and photoautotroph, respectively. The accuracy and effectiveness of the proposed protocol was demonstrated using two model coculture pairs, Methylomicrobium alcaliphilum 20ZR - Synechococcus sp. PCC7002 that prefers high pH high salt condition, and Methylococcus capsulatus - Chlorella sorokiniana that prefers low salt and neutral pH medium. The performance of the proposed protocol was compared with a flow cytometry based cell counting approach. The experimental results show that the proposed protocol is much easier to carry out and delivers faster and more accurate results in measuring individual biomass concentration than the cell counting approach without requiring any special equipment.
\end{abstract}

\section{Introduction}

Biogas is comprised primarily of methane $\left(\mathrm{CH}_{4}, 50 \% \sim 70 \%\right)$ and carbon dioxide $\left(\mathrm{CO}_{2}, 30 \% \sim 50 \%\right)$. It can be produced through anaerobic digestion (AD) of various organic waste sources, including landfill waste; animal manure; wastewater sludge; and industrial, institutional, and commercial organic wastes. $\mathrm{CO}_{2}$ and $\mathrm{CH}_{4}$ are the two leading greenhouse gases (GHGs) that cause many detrimental effects to our ecosystem, including climate change. On the other hand, $\mathrm{CH}_{4}$ is also a valuable fuel. It is estimated that currently US biogas production potential is 654 billion cubic feet per year, which could displace 7.5 billion gallon of gasoline (AgSTAR, 2018). Although waste-derived biogas has immense potential as a renewable feedstock for producing high-density fuels and commodity chemicals, the contaminants (e.g., $\mathrm{H}_{2} \mathrm{~S}, \mathrm{NH}_{3}$, and volatile organic carbon (VOC) compounds) present significant challenges to biogas utilization. Currently the ADderived biogas is primarily used for heating/cooking or flared, with only a small fraction for electricity generation due the cost associated with biogas cleanup (AgSTAR, 2018). To tap into this immense potential, effective technologies that can co-utilize both $\mathrm{CO}_{2}$ and $\mathrm{CH}_{4}$ without costly biogas cleanup are needed.

Recent studies have demonstrated that natural microbial communities have developed a highly efficient way to recover the energy and capture carbon from natural biogas streams through interspecies metabolic coupling of methane oxidation to oxygenic photosynthesis (Kip et al., 2010; Milucka et al., 2015; Raghoebarsing et al., 2005). Figure 1(a) illustrates the key synergistic interactions within the methanotroph-photoautotroph coculture: the photoautotroph converts $\mathrm{CO}_{2}$ into biomass while producing $\mathrm{O}_{2}$ via photosynthesis and the methanotroph utilizes the in situ produced $\mathrm{O}_{2}$ to convert $\mathrm{CH}_{4}$ into biomass while producing $\mathrm{CO}_{2}$ for the photoautotroph. Figure 1(b) depicts the total mass balance and key substrate exchanges in the coculture. 
Following the principles that drive the natural consortia, different synthetic methanotroph-photoautotroph (e.g., cyanobacteria or microalgae) cocultures have been demonstrated to simultaneously convert both $\mathrm{CH}_{4}$ and $\mathrm{CO}_{2}$ into microbial biomass without external oxygen supply (Badr, Hilliard, Roberts, He, \& Wang, 2019; Hill, Chrisler, Beliaev, \& Bernstein, 2017; Rasouli, Valverde-Pérez, D’Este, De Francisci, \& Angelidaki, 2018; van der Ha et al., 2012; Wang \& He, 2018). The biogas-derived coculture biomass could be further processed to produce biofuels (such as biodiesel), directly used as single cell protein for animal feed supplement, or serves as feedstock to produce bioplastics. In addition, the coculture could be engineered to produce other valueadded chemicals (such as succinate or lactic acid) using biogas as feedstock. Therefore, the methanotrophphotoautotroph coculture offers a highly promising biological platform for waste-to-value conversion.

In order to develop methanotroph-photoautotroph based biotechnology for biogas conversion, a key prerequisite is an effective tool to enable fast, easy and accurate characterization of each organism in the coculture in terms of biomass growth and biogas conversion performance. However, currently no such tool is available. In fact, one major challenge associated with characterizing any mixed culture is the accurate determination of the individual biomass concentration for each microorganism. Existing approaches to quantify individual biomass concentration in mixed culture include molecular biological, biochemical, and microbiological method (Sabra, Dietz, Tjahjasari, \& Zeng, 2010; Spiegelman, Whissell, \& Greer, 2005). However, these methods require either expensive equipment such as flow cytometry, community genome sequencing, or time-consuming and challenging techniques, such as RNA/DNA extraction, isolation, or amplification. Therefore, these approaches are suitable for off-line, infrequent characterization of mixed culture, and cannot provide the frequent or real-time measurements desired for dynamic modelling of the coculture systems. As a result, among the published methanotroph-photoautotroph research, only Hill et al. (2017) tracked the individual biomass concentration over time through cell counting using flow cytometry, while others just reported the total optical density of the coculture over time without differentiating the contribution from the methanotroph and the photoautotroph (Rasouli et al., 2018; van der Ha et al., 2012).

Besides individual biomass concentration, the individual substrate consumption rates and product excretion rates of each organism are needed in order to develop a kinetic model for the coculture. However, when there is cross-feeding in the coculture (i.e., any exchange of metabolite(s) between different organisms), it is highly challenging to obtain the individual consumption/production rates because they cannot be measured directly. For the case of methanotroph-photoautotroph coculture, as shown in Figure 1(b), both $\mathrm{O}_{2}$ and $\mathrm{CO}_{2}$ are cross-feeding metabolites: $\mathrm{O}_{2}$ is produced by the photoautotroph while consumed by the methanotroph, while $\mathrm{CO}_{2}$ is produced by the methanotroph and consumed by the photoautotroph. However, what can be directly measured are the overall or total consumption/production rates of $\mathrm{O}_{2}$ and $\mathrm{CO}_{2}$ by the coculture, not individual rates by each organism. Currently how to use the measured overall rate to infer or estimate the individual consumption/production rates remains an unsolved problem. It is worth noting that in our experiments, oftentimes no oxygen was detectable in the gas phase or liquid phase, as all the oxygen produced by the photoautotroph was consumed by the methanotrophin situ .

To address the above mentioned challenges, we have developed an experimental-computational (E-C) protocol to fully characterize the synthetic methanotroph-photoautotroph coculture based on the overall mass balance and each organism's growth stoichiometry. Besides tracking the biomass concentration of each organism in the coculture over time, the E-C protocol also obtains estimates on the substrate consumption rates $\left(\mathrm{CH}_{4}\right.$ and $\mathrm{O}_{2}$ uptake rates for the methanotroph and $\mathrm{CO}_{2}$ uptake rate for the photoautotroph) and product secretion rates $\left(\mathrm{CO}_{2}\right.$ for the methanotroph and $\mathrm{O}_{2}$ for the photoautotroph). Such quantitative characterizations will enable better understanding of the coculture growth kinetics, and will lay the foundation for the development of the coculture-based biotechnology to convert biogas into valuable products. The E-C protocol only requires the commonly measured variables including total optical density for the coculture (UV/Vis spectroscopy), gas phase composition (GC), dissolved $\mathrm{CO}_{2}$ in the culture broth (total carbon analyser). Therefore, the E-C protocol does not require any special equipment, and it does not require any special sample preparation such as DNA/RNA extraction or cell fixation in order to achieve the above-mentioned characterizations.

In this work, we use one methanotroph-cyanobacteria pair and one methanotroph-microalgae pair to de- 
monstrate the performance of the developed protocol; To validate its accuracy, we compared the individual biomass concentrations obtained by the E-C protocol with cell counting results obtained using flow cytometry. In this work, the methanotroph-cyanobacteria coculture pair is Methylomicrobium alcaliphilum 20ZR Synechococcus sp. PCC7002, which prefers high salt high pH medium and has demonstrated robust growth on different concentrations of biogas (Hill et al., 2017). The methanotroph-microalgae coculture pair is $M e$ thylococcus capsulatus - Chlorella sorokiniana, which prefers low salt and neutral $\mathrm{pH}$ medium and has been used for wastewater treatment (Rasouli et al., 2018).

\section{Materials and Methods}

Microorganism and growth media

Methylomicrobium alcaliphilum 20ZR was provided by Dr. Marina Kalyuzhnaya, San Diego State University, and Synechococcus sp. PCC7002 was provided by Dr. Alexander Beliaev, Pacific Northwest National Lab. M. alcaliphilum 20ZR cells were grown in modified P-medium.Synechococcus sp. PCC7002 cells were grown in $\mathrm{A}+$ medium. For the coculture, the growth media consisted of $10 \% \mathrm{P}$-medium and $90 \% \mathrm{~A}+\mathrm{medium}$.

Methylococcus capsulatuswas acquired from the American Type Culture Collection (ATCC 33009), and Chlorella sorokiniana (UTEX 2805) was acquired from UTEX Culture Collection of Algae. M. capsulatus Bath cells were grown in NMS medium. C. sorokiniana cells were grown in N8 medium. For the coculture, the growth media consisted of $10 \%$ NMS medium and $90 \%$ N8 medium.

Sampling procedure

Composition of gas samples was analysed using GC (Agilent 7890B gas chromatograph customized with FID, TCD, Unibeads IS 60/80 mesh and MolSieve 5A 60/80 SST columns). It is worth noting that the consumption and production of various gases will result in changes in system pressure (for batch operations) or off-gas flow rate (for continuous operations). These changes would cause significant errors in the estimated gas component uptake and production rates if they were not accounted for. To address it, we have previously developed a protocol (Stone, He, \& Wang, 2019), which is followed in this work. The optical density of each liquid sample was measured using a Beckman Coulter DU ${ }^{\circledR} 730 \mathrm{UV} / \mathrm{V}$ is spectrophotometer at OD750. To determine the amount of the dissolved $\mathrm{CO}_{2}$ in the liquid phase, we first removed cell mass through

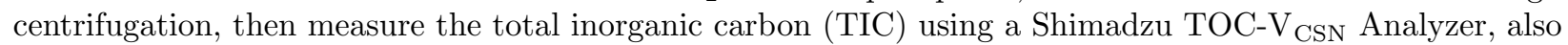
following the procedures reported in (Stone et al., 2019).

Cell counting through flow cytometry

For M. alcaliphilum 20ZR - S. sp. PCC7002 pair, two $0.5 \mathrm{~mL}$ samples of culture broth were taken and each sample was immediately treated with $0.25 \mathrm{~mL}$ of $50 \mathrm{mM}$ EDTA and $0.25 \mathrm{~mL}$ of $4 \%$ paraformaldehyde to fix the cells. After 10 minutes of fixation, the samples were centrifuged at 10,000 RPM and 0.5 ml of supernatant was removed, then each sample was treated with $0.5 \mathrm{~mL}$ of $0.05 \%$ Tween-20 detergent for 20 minutes (away from light) to minimize cells sticking to each other. Next, after removing Tween-20 through centrifugation, the samples were washed and re-suspended in DI water. For M.capsulatus - C. sorokiniana pair, the overall procedure is similar, with the differences being that the samples were first treated with $0.2 \%$ Tween-20 detergent for 20 minutes and then treated with $200 \mathrm{mM}$ EDTA and $4 \%$ paraformaldehyde for 20 minutes to fix the cells. After sample preparation, $25 \mu \mathrm{L}$ of the re-suspended sample was counted on a Beckman Coulter Cytoflex LX cytometer with 6 active lasers and 21 channels for fluorescence detection. FlowJo Version 10.6 .1 was used to analyze the data obtained from the flow cytometer. As both the cyanobacteria and microalgae used in this work are green, and both methanotrophs are white, different filter of excitation wavelengths were used to help differentiate the cells in the coculture. For M.alcaliphilum 20ZR - S. sp. PCC7002 pair, the forward scatter (FSC-H) was paired with the filter of excitation wavelength at 610nm (Y610-mCHERRY-H fluorochrome) to separate the two populations. For $M$. capsulatus - C. sorokiniana pair, the FSC-H was paired with the filter of excitation wavelength of 710nm (Y710-PC5.5-H) to separate the two populations.

Calibration and testing for the cell counting approach 
To establish the calibration relationship between biomass concentration and cell counting results, we first conducted cell counting experiments for each single culture (M. alcaliphilum 20ZR, S. sp.PCC7002, M. capsulatus and C. sorokiniana). For each strain, cell counting was performed for 4 different biomass concentrations, with triplicates for each sample. To validate the effectiveness of the cell counting approach, we prepared static coculture samples by mixing given amounts of each individual microorganisms together. For each coculture pair, six coculture samples with different compositions were tested with triplicates.

Demonstration of the E-C protocol in characterizing coculture dynamic growth

In these experiments, the E-C protocol was applied to characterize the dynamic growth of both model coculture pairs. To validate the E-C protocol's accuracy, the individual biomass concentration within the coculture was also measured through cell counting using flow cytometry for comparison. For each coculture pair, three different inoculum concentrations were tested with duplicates. For the M. alcaliphilum 20ZR - S. sp. PCC7002 pair, the inoculum OD ratios between the methanotroph and the cyanobacteria were 1:15, 1:10, and 1:5, with the same amount of methanotroph for all three cases. For theM. capsulatus - $C$. sorokiniana pair, the inoculum OD ratios were 1:3, 1:2 and 1:1, also with the same amount of methanotroph for all three cases. Before and after the inoculation, all vials were flushed with the feeding gas $\left(80 \% \mathrm{CH}_{4}\right.$ and $\left.20 \% \mathrm{CO}_{2}\right)$, and were put under the same light intensity $\left(190 \mu \mathrm{mol} / \mathrm{m}^{2} / \mathrm{s}\right)$. The coculture growth lasted for 3 days and was sampled once daily. The vials were flushed with feeding gas to replenish the gas phase after each sampling.

\section{Modeling Framework for the Experimental-Computational Protocol}

The protocol was developed based on each organism's growth stoichiometry, the substrate exchange relationship within the coculture as shown in Figure 1(b), and the total mass balance. Eqns. (1) and (2) show the growth stoichiometry for the methanotroph and photoautotroph, respectively.

$$
\begin{aligned}
& \mathrm{CH}_{4}+\left(Y_{\frac{\mathrm{O}_{2}}{C \mathrm{C}_{4}}}\right)_{\text {meth }} \mathrm{O}_{2} \rightarrow\left(Y_{\frac{X}{\mathrm{CH}_{4}}}\right)_{\text {meth }} X_{\text {meth }}+\left(Y_{\frac{\mathrm{CO}_{2}}{\mathrm{CH}_{4}}}\right)_{\text {meth }} \mathrm{CO}_{2}(1) \\
& \mathrm{CO}_{2}+\left(Y_{\frac{\mathrm{H}_{2} \mathrm{O}}{\mathrm{CO}}}\right)_{\text {photo }} \mathrm{H}_{2} \mathrm{O} \rightarrow\left(Y_{\frac{X}{C \mathrm{C}_{2}}}\right)_{\text {photo }} X_{\text {photo }}+\left(Y_{\frac{\mathrm{O}_{2}}{\mathrm{CO}_{2}}}\right)_{\text {photo }} \mathrm{O}_{2}(2)
\end{aligned}
$$

where $X$ denotes biomass, and the subscripts "meth" and "photo" denote methanotroph and photoautotroph, respectively; $Y_{\frac{a}{b}}$ denotes the stoichiometric coefficients between " $a$ " and " $b$ ", where " $b$ " is $\mathrm{CH}_{4}$ for methanotroph and $\mathrm{CO}_{2}$ for photoautotroph. These coefficients can be obtained from literature (Akberdin et al., 2018; Bernstein et al., 2016; Kliphuis et al., 2011). If the coculture growth medium is vastly different from what is commonly used for the single culture and could affect the microorganism's growth stoichiometry, then experimental data of the single culture cultivated on the coculture medium should be used to estimate the coefficients. The coefficients used in this work are listed in Table 1.

As shown in Figure 1 (b), only the methanotroph within the coculture can consume $\mathrm{CH}_{4}$, therefore the amount of cell growth for methanotroph can be estimated based on the measured methane consumption (i.e., $\mathrm{CH}_{4}$ ). Similarly, the amount of the $\mathrm{O}_{2}$ required for methane consumption and the amount of $\mathrm{CO}_{2}$ produced can be estimated using stoichiometric coefficients as follows.

$$
\begin{aligned}
& (X)_{\text {meth }}=\left(Y_{\frac{X}{C H_{4}}}\right)_{\text {meth }} \mathrm{CH}_{4}(3) \\
& \left(\mathrm{O}_{2}\right)_{\text {meth }}=\left(Y_{\frac{\mathrm{O}_{2}}{\mathrm{CH}_{4}}}\right)_{\text {meth }} \mathrm{CH}_{4}(4) \\
& \left(\mathrm{CO}_{2}\right)_{\text {meth }}=\left(Y_{\frac{\mathrm{CO}_{2}}{\mathrm{CH}_{4}}}\right)_{\text {meth }} \mathrm{CH}_{4}(5)
\end{aligned}
$$

Next, based on the overall mass balance of $\mathrm{O}_{2}$ and $\mathrm{CO}_{2}$, as shown in Eqns (6) and (7), we can determine the amount of $\mathrm{CO}_{2}$ consumed and the amount of $\mathrm{O}_{2}$ produced by the photoautotroph. The subscript "gas" 
and "liquid" denote the measurements obtained from headspace samples and liquid samples, respectively.

$$
\begin{aligned}
& \left(\mathrm{O}_{2}\right)_{\text {gas }}=\left(\mathrm{O}_{2}\right)_{\text {photo }}-\left(\mathrm{O}_{2}\right)_{\text {meth }}(6) \\
& \left(\mathrm{CO}_{2}\right)_{\text {gas }}=\left(\mathrm{CO}_{2}\right)_{\text {meth }}-\left(\mathrm{CO}_{2}\right)_{\text {photo }}-\left(\mathrm{CO}_{2}\right)_{\text {liquid }}
\end{aligned}
$$

where $\mathrm{CO}_{2}$ and $\mathrm{O}_{2}$ in the gas phase (i.e., $\left.\left(\mathrm{CO}_{2}\right)_{\text {gas }},\left(\mathrm{O}_{2}\right)_{\text {gas }}\right)$ are measured through $\mathrm{GC}$, and the dissolved $\mathrm{CO}_{2}$ in the liquid phase (i.e. , $\left.\left(\mathrm{CO}_{2}\right)_{\text {liquid }}\right)$ are measured through total carbon analyser. In Eqn (6), we neglect the contribution from dissolved $\mathrm{O}_{2}$ due to its small solubility in aqueous solutions; however, in Eqn. (7), dissolved $\mathrm{CO}_{2}$ has to be considered due to its much larger solubility in aqueous solutions, especially under high $\mathrm{pH}$ conditions. Although it is difficult to determine the amount of dissolved $\mathrm{CO}_{2}$ in one sample due to the carbonate $\left(\mathrm{CO}_{3}^{2-}\right)$ and bicarbonate $\left(\mathrm{HCO}_{3}^{-}\right)$salts contained in the culture medium and the equilibrium among different forms of dissolved $\mathrm{CO}_{2}$, the change in dissolved $\mathrm{CO}_{2}$ between two sampling points can be easily determined by the difference in the total inorganic carbon content of these two samples. Therefore, based on the overall mass balances (i.e., Eqns (6) and (7)), the amount of $\mathrm{CO}_{2}$ consumed and $\mathrm{O}_{2}$ produced by photoautotroph can be obtained, as shown in Eqns (8) and (9).

$$
\begin{aligned}
& \left(\mathrm{O}_{2}\right)_{\text {photo }}=\left(\mathrm{O}_{2}\right)_{\text {gas }}+\left(\mathrm{O}_{2}\right)_{\text {meth }}(8) \\
& \left(\mathrm{CO}_{2}\right)_{\text {photo }}=\left(\mathrm{CO}_{2}\right)_{\text {meth }}-\left(\mathrm{CO}_{2}\right)_{\text {gas }}-\left(\mathrm{CO}_{2}\right)_{\text {liquid }}
\end{aligned}
$$

With the amount of $\mathrm{CO}_{2}$ consumed and $\mathrm{O}_{2}$ produced by the photoautotroph available, the amount of biomass produced by photoautotroph growth can be obtained through two ways using growth stoichiometry, either from $\mathrm{CO}_{2}$ consumption (Eqn. (10)) or from $\mathrm{O}_{2}$ production (Eqn. (11)).

$$
\begin{aligned}
& (X)_{\text {photo-1 }}=\left(Y_{\frac{X}{\mathrm{CO}_{2}}}\right)_{\text {photo }}\left(\mathrm{CO}_{2}\right)_{\text {photo }}(10) \\
& (X)_{\text {photo-2 }}=\left(Y_{\frac{X}{\mathrm{O}_{2}}}\right)_{\text {photo }}\left(O_{2}\right)_{\text {photo }}(11)
\end{aligned}
$$

where biomass yield with respect to $\mathrm{O}_{2}$ can be obtained as the following:

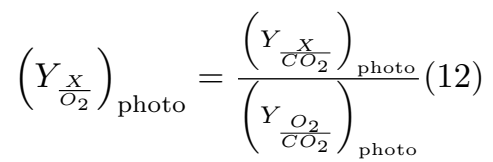

In this work, we use the average of these two approaches to estimate photoautotroph biomass accumulation, as shown Eqn. (13).

$$
(X)_{\text {photo }}=\frac{1}{2}\left[(X)_{\text {photo-1 }}+(X)_{\text {photo- } 2}\right](23)
$$

\section{Results and Discussion}

\subsection{Calibration and validation of the cell counting approach}

The flow cytometry calibration plots for each microorganism are given in the supplementary material Figure S1. These results confirmed excellent linear relationship between biomass concentration and the cell counting results, with $\mathrm{R}^{2}$ ranging $0.979-0.983$. Figure $\mathrm{S} 1$ also shows that cell counting with flow cytometry is more reliable/consistent when cell concentrations are low. Using the calibration relationship obtained from the single cultures, we validated the accuracy of the flow cytometry measurements using static coculture samples with known individual biomass concentrations. For each coculture pair, six samples with different compositions were tested.

The individual biomass concentrations for each microorganism in the cocultures measured using flow cytometry are plotted against the known concentrations in Figure 2 (a) (d), with the detailed results provided in Supplementary Material Table S1. As shown in these figures, the measured individual biomass concentrations (converted from cell counting based on the calibration curves in Figure S1) show good agreement with the known concentrations. However, there are relatively large variations among the triplicates for each sample, 
especially for higher concentrations, which is consistent with the similar trend observed in the calibration curves in Figure S1. In addition, when the same sample was measured multiple times, the measurements showed same level of variations, suggesting the source of the variation was cell counting. One possible reason for such large variation is the non-uniform distribution of the cells in the liquid sample, and the small sample volumes $(25 \mu \mathrm{L})$ for cell counting makes such variation more pronounced for higher concentrations, as observed in both calibration and validation experiments. Another possible reason, which we believe is more important for mixed culture samples, is the effect of sample fixation process on cell counting.

In this work, we had to optimize the sample fixation protocols multiple times in order to obtain the acceptable validation results. Figure 2 (e) and (f) compare the cell counting results for a same static coculture sample. Figure 2 (e) was obtained following the cell fixation protocol initially developed for the salt water pair, while Figure 2 (f) following the protocol optimized for the fresh water pair. The known and measured individual biomass concentration are provided in Table 2. The large measurement errors shown in Figure 2 (e) (-88.3\% for the methanotroph and $18.0 \%$ for the microalgae) indicate that some methanotroph cells stuck to the microalgae cells and the flow cytometer could not separate them properly. With the optimized protocol, methanotroph cells were much better separated from microalgae cells, which resulted in significantly reduced measurement error (-7.9\% for the methanotroph and $-6.5 \%$ for the microalgae). Currently the flow cytometry has been commonly used to characterize the composition of synthetic microbiome. This example highlights the importance of performing validation experiments to confirm the appropriateness of the experimental protocol and the accuracy of the cell counting to avoid misleading results.

Validation of E-C protocol using dynamic growth of the coculture

The E-C protocol is not applicable to the static coculture with known concentrations, as it is based on the growth stoichiometry of individual microorganisms. Therefore, in this subsection, we use coculture batch growth experiments to demonstrate and validate the E-C protocol. With the validity of the cell counting method established, the individual biomass concentration obtained from the cell counting method were used to validate the E-C protocol. Figure 3 (a) and (b) plot the total OD of the coculture over 3 days for the salt water pair and fresh water pair, respectively; and Figure 3 (c) and (d) plot the gas phase composition for each coculture pair for one inoculum ratio (1:10 for the salt water pair and 1:2 for the fresh water pair), respectively. The gas compositions for the other inoculum ratios are provided in Supplementary Material Figure S2. For the fresh water methanotroph-microalgae pair, higher inoculum concentration of the microalgae resulted in better growth of the coculture. This is because the microalgae grows much slower than the methanotroph, so the methanotroph growth is limited by $\mathrm{O}_{2}$ availability. Therefore, more microalgae in the inoculum enabled better growth of the methanotroph. For the salt water pair, higher inoculation concentration of the cyanobacteria did not have much impact on coculture growth. This is because the cyanobacteria grew much faster than the methanotroph, and the methanotroph growth is limited by mass transfer of $\mathrm{CH}_{4}$ from gas phase.

Figure 4 compares the individual biomass concentration measured through the cell counting approach and the E-C protocol for both coculture pairs, where each point represents one of the duplicates, and the error bar represents the standard deviation from three cell counting measurements for the same sample. As can be seen from these figures, the results obtained from the two approaches correlated very well, particularly at low biomass concentrations. The $\mathrm{R}^{2}$ for the linear relationship between the results from the E-C protocol and cell counting approach ranges $0.90-0.98$, which validates the results obtained from the E-C protocol.

However, Figure 4 also shows that the agreement between the cell counting approach and the E-C protocol deteriorates at higher concentrations after coculture growth. To determine which approach performs better, we calculated the total OD for each sample using the measured individual biomass concentrations, and plotted them against the measured total OD. The results are shown in Figure 5 (a) and (b) for the salt water pair and the fresh water pair respectively. Both figures showed that the total OD calculated from the E-C protocol were almost exactly the same as the measured total OD. On the other hand, the total OD calculated from the cell counting approach showed larger deviation from the measured total OD, particularly at higher concentrations. The bar chart of the mean squared error (MSE) of predictions in the total OD based on six 
experimental runs (three inoculum concentrations with duplicates) are plotted in Figure 5. The error bar represents one standard deviation of MSE's. Student's $t$-test shows that the MSE's of the cell counting is statistically significantly larger than that of the E-C protocol, with a p-value of 0.0158 for the salt water pair and 0.0030 for the fresh water pair.

Besides obtaining individual biomass concentration for each microorganism in the coculture accurately and quickly, the E-C protocol also provides estimates of individual substrate consumption rates and product excretion rates. Figure 6 (a) and (b) plot the individual consumption and production rates of $\mathrm{O}_{2}$ and $\mathrm{CO}_{2}$ respectively by $M$. alcaliphilum 20ZR and $S$. sp. PCC7002 over a three-day period for the inoculum ratio of 1:10, and Figure 6 (c) and (d) plot those values for M.capsulatus - C. sorokiniana, for the inoculum ratio of $1: 2$.

Figure 6 shows that although for many cases very small amounts of $\mathrm{O}_{2}$ were detected in the gas phase (e.g., day 2 and 3 for the salt water pair and all 3 days for the fresh water pair), significant amount of $\mathrm{O}_{2}$ was produced by the photoautotroph, which was completely consumed in situ by the methanotroph. Similarly, Figure 6 shows that the actual amount of $\mathrm{CO}_{2}$ consumed by the photoautotroph was much larger than what was directly measured in the experiment, because the $\mathrm{CO}_{2}$ produced by the methanotroph would be preferably consumed by the photoautotroph, as it was produced in situ and did not involve the mass transfer resistance from gas to liquid.

\section{Conclusions}

It has been recognized that methanotroph-photoautotroph cocultures offer a highly promising biological platform for biogas conversion. Through the interspecies metabolic coupling of methane oxidation to oxygenic photosynthesis, the coculture can simultaneously convert both $\mathrm{CH}_{4}$ and $\mathrm{CO}_{2}$ into microbial biomass without external oxygen supply. However, one key obstacle in developing methanotroph-photoautotroph based biotechnology for biogas conversion is the lack of an effective tool for fast, accurate and frequent characterization of the coculture growth dynamics. In this work, based on the organism's growth stoichiometry, the interspecies metabolic coupling and the total mass balance, we developed an E-C protocol to characterize the coculture. The E-C protocol provides not only accurate estimates of the individual biomass concentration within the coculture, but also the individual substrate consumption and product excretion rates of each organism. To the best of our knowledge, the developed E-C protocol is the first ever approach that could obtain individual substrate consumption and product excretion rates for methanotroph-photoautotroph or any other cocultures.

The accuracy of the developed E-C protocol was validated by cell counting approach using flow cytometry. In addition, by comparing the predicted total OD from the individual biomass concentration with the measured total OD, we showed that the E-C protocol provided better accuracy than the cell counting approach through statistical testing. It is worth noting that the developed E-C protocol only requires commonly used analytical equipment to provide quick and accurate characterization of the methanotroph-photoautotroph cocultures.

Finally, we showed that it is very important to use static cocultures with known concentration to validate the cell counting method, as the cell fixation protocol could result in severely skewed cell counting results. Currently, although cell counting with flow cytometry has become increasingly common in determining the individual biomass concentration in mixed culture or microbiome, very few publications have presented validation results on their cell counting approaches.

\section{Acknowledgements}

This work was supported by U.S. Department of Energy, Office of Science, Office of Biological and Environmental Research, Genomic Science Program (DE-SC0019181), and Department of Education (P200A180002). The authors declare that there is no conflict of interest.

\section{Reference}

AgSTAR, U. S. (2018). Market opportunities for biogas recovery systems at U.S. livestock facilities. 
Akberdin, I. R., Thompson, M., Hamilton, R., Desai, N., Alexander, D., Henard, C. A., .. Kalyuzhnaya, M. G. (2018). Methane utilization in Methylomicrobium alcaliphilum 20Z R: a systems approach.Scientific Reports , 8 (1), 2512.

Badr, K., Hilliard, M., Roberts, N., He, Q. P., \& Wang, J. (2019). Photoautotroph-Methanotroph Coculture - A Flexible Platform for Efficient Biological CO2-CH4 Co-utilization. IFAC-PapersOnLine ,52 (1), 916921. https://doi.org/10.1016/j.ifacol.2019.06.179

Bernstein, H. C., McClure, R. S., Hill, E. A., Markillie, L. M., Chrisler, W. B., Romine, M. F., .. others. (2016). Unlocking the constraints of cyanobacterial productivity: acclimations enabling ultrafast growth. MBio , 7 (4), 949.

Hill, E. A., Chrisler, W. B., Beliaev, A. S., \& Bernstein, H. C. (2017). A flexible microbial co-culture platform for simultaneous utilization of methane and carbon dioxide from gas feedstocks. Bioresource Technology .

Kip, N., van Winden, J. F., Pan, Y., Bodrossy, L., Reichart, G.-J., Smolders, A. J. P., ... den Camp, H. J. M. O. (2010). Global prevalence of methane oxidation by symbiotic bacteria in peat-moss ecosystems. Nature Geoscience , 3 (9), 617-621.

Kliphuis, A. M. J., Janssen, M., van den End, E. J., Martens, D. E., \& Wijffels, R. H. (2011). Light respiration in Chlorella sorokiniana.Journal of Applied Phycology , 23 (6), 935-947.

Milucka, J., Kirf, M., Lu, L., Krupke, A., Lam, P., Littmann, S., .. Schubert, C. J. (2015). Methane oxidation coupled to oxygenic photosynthesis in anoxic waters. The ISME Journal .

Raghoebarsing, A. A., Smolders, A. J. P., Schmid, M. C., Rijpstra, W. I. C., Wolters-Arts, M., Derksen, J., ... others. (2005). Methanotrophic symbionts provide carbon for photosynthesis in peat bogs. Nature , 436 (7054), 1153-1156.

Rasouli, Z., Valverde-Pérez, B., D'Este, M., De Francisci, D., \& Angelidaki, I. (2018). Nutrient recovery from industrial wastewater as single cell protein by a co-culture of green microalgae and methanotrophs. Biochemical Engineering Journal , 134, 129-135.

Sabra, W., Dietz, D., Tjahjasari, D., \& Zeng, A.-P. (2010). Biosystems analysis and engineering of microbial consortia for industrial biotechnology. Engineering in Life Sciences , 10 (5), 407-421. Retrieved from http://pbi.hospedagemdesites.ws/wp-content/uploads/2012/12/Biosystems-analysis-and-engineeringof-microvial-consortia-for-industrial-biotechnology.pdf

Spiegelman, D., Whissell, G., \& Greer, C. W. (2005). A survey of the methods for the characterization of microbial consortia and communities. Canadian Journal of Microbiology , 51 (5), 355-386. Retrieved from http://www.researchgate.net/profile/Dan_Spiegelman/publication/7672619_A_survey_of_the_methods_for_the_characterization_of_microbial_consortia_and_communities/links/0046353ac3ffbc37c5000000.pdf

Stone, K. A., He, Q. P., \& Wang, J. (2019). Two Experimental Protocols for Accurate Measurement of Gas Component Uptake and Production Rates in Bioconversion Processes. Scientific Reports , 9 (1), 5899. https://doi.org/10.1038/s41598-019-42469-3

van der Ha, D., Nachtergaele, L., Kerckhof, F.-M., Rameiyanti, D., Bossier, P., Verstraete, W., \& Boon, N. (2012). Conversion of biogas to bioproducts by algae and methane oxidizing bacteria. Environmental Science \& Technology , 46 (24), 13425-13431.

Wang, J., \& He, Q. P. (2018). Methanotroph-microalgae coculture. US Provisional Patent Application $62 / 664,565$.

\section{Hosted file}

Tables v2.docx available at https://authorea.com/users/349734/articles/474693-fast-and-easyquantitative-characterization-of-methanotroph-photoautotroph-cocultures 